\title{
IAP ALS Update on Resuscitation Guidelines During COVID-19 Pandemic
}

\author{
Lokesh Tiwari $^{1,2}$ (D) $\cdot$ L N Taneja ${ }^{2,3} \cdot$ Shalu Gupta ${ }^{4} \cdot$ Other Expert Committee Members
}

Received: 31 May 2020 / Accepted: 24 August 2020 / Published online: 27 September 2020

(C) Dr. K C Chaudhuri Foundation 2020

\begin{abstract}
Corona virus disease 2019 (COVID-19) pandemic has posed significant risk for health care workers. Various steps of cardiopulmonary resuscitation involve aerosol-generating procedures and have significant risk of spread of corona virus. Indian Academy of Pediatrics Advanced Life Support Basic Life Support (IAP ALS BLS) group had constituted a guideline update team to suggest modifications in existing resuscitation guidelines in view of COVID-19 pandemic. The GRADE approach was used to develop recommendations on shortlisted clinical practice questions on resuscitation during COVID pandemic as modifications.
\end{abstract}

Keywords Resuscitation · IAP ALS BLS · COVID-19

\section{Introduction}

Corona virus disease 2019 (COVID 19) pandemic has posed significant risk and new challenges for health care workers (HCWs) due to highly contagious nature of the virus. Steps of cardiopulmonary resuscitation (CPR) may further expose the

Expert Committee Members: Lokesh Tiwari, Department of Pediatrics, All India Institute of Medical Sciences, Patna, Bihar, India; National Coordinator IAP BLS Group; L N Taneja, Department of Pediatrics, Max Super Specialty Hospital, IP Extension, Delhi, India; Chairperson IAP BLS Group; Shalu Gupta, Department of Pediatrics, Lady Hardinge Medical College and Kalawati Saran Children Hospital, New Delhi, India; A K Sharma, National Convenor IAP ALS Group; Narendra Nanivadekar, Joint National Convenor IAP ALS Group; Sudhakar Gajjala, Department of Pediatrics, KIMS Hospitals Kurnool, India; Vice President (Central Zone) CIAP; Janani Sankar, Kanchi Kamkoti Child Trust Hospital, Chennai, India; Maninder Singh Dhaliwal, Pediatric Critical Care Unit, Department of Pediatrics, Medanta, The Medicity Gurugram, India; Santanu Deb, Department of Pediatrics, Nazareth Hospital, Shillong, India

Lokesh Tiwari

lokeshdoc@yahoo.com

1 Pediatric Intensive Care Unit, Department of Pediatrics, All India Institute of Medical Sciences, Patna, Bihar, India

2 IAP BLS Group, New Delhi, India

3 Department of Pediatrics, Max Super Specialty Hospital, IP Extension, Delhi, India

4 Department of Pediatrics, Lady Hardinge Medical College and Kalawati Saran Children Hospital, New Delhi, India
HCWs to infection due to aerosol generation $[1,2]$. Understanding the urgent need of modifications in existing resuscitation guidelines [3, 4], Indian Academy of Pediatrics Advanced Life Support Basic Life Support (IAP ALS BLS) group has formulated ten recommendations on the general principles for resuscitation to be followed during COVID-19 pandemic. Recommendations are formulated considering various measures for better protection of HCWs, prioritizing oxygenation and ventilation methods with lower risk of aerosolization, addition of magnesium sulphate in crash cart and early start of vasoactive drugs and appropriateness of starting and continuing resuscitation. These guidelines are evolving, and modifications are expected as we continue to understand COVID-19 [5].

\section{Material and Methods}

Understanding the urgent need of modifications in resuscitation guidelines, IAP ALS BLS group had constituted a guideline update team comprising of experts in the field of pediatric resuscitation and involved in managing patients with COVID-19 to provide an update and clinical practice guidelines on resuscitation during COVID pandemic. The update team drafted a list of questions which are likely to be faced by pediatricians and other health care workers while providing resuscitation to a child with cardiac arrest during COVID pandemic. Questions were circulated among experts and prioritization sequence was decided after rounds of video conferences. Literature search was carried out in PubMed combining the 
search term ("COVID-19" [All Fields] OR "coronavirus" [All Fields]) AND 2019/12 [PDAT]: OR 2019nCoV [All Fields] OR SARS-CoV-2 [All Fields]) with other key words relevant to the practice question being addressed. Additionally, websites of the relevant professional organizations were also searched. Guidelines, systematic reviews, trials, narrative reviews and other descriptive reports were reviewed. The context, resources required, values and preferences were considered before recommending necessary modifications in existing guidelines. Final recommendations were made on consensus among all experts through exchange of drafts via emails and video based conference meetings.

The objective of this update is to provide necessary modifications in existing IAP CPR guidelines on the shortlisted clinical practice questions (Box 1).

\section{Box 1. Shortlisted clinical practice questions}

1. What protective measures should be undertaken before starting chest compression in COVID suspected or confirmed victim?

2. What additional measures should be taken while securing airway of the victim?

3. What should be the team composition to minimize the risk of transmission of infection without compromising the quality of CPR?

4. What are additional steps to reduce the chances of COVID exposure to health care worker while placing tracheal tube and providing positive pressure ventilation?

5. Is AED comparable to manual defibrillator while delivering shock during COVID-19 pandemic considering safety, cost, access and training in Indian context?

6. Does CPR in prone position outweighs the risk of complications of turning supine and providing CPR in a COVID-19 positive cardiac arrest victim who is already being ventilated in prone position?

7. Is addition of any drug in resuscitation cart or modification in timing of vasoactive drugs likely to improve outcome in the context of COVID disease and existing treatment recommendations for the same?

8. What are the available resources to help while deciding reasonability of starting CPR and its duration?

COVID-19 Corona virus disease 2019; CPR Cardiopulmonary resuscitation

\section{Ten Recommendations for CPR during COVID-19 Pandemic}

\section{Protect Yourself Before Starting Chest Compression: (P-C-A-B Sequence)}

- HCW should don full personal protective equipment (PPE) while handling In Hospital Cardiac Arrest (IHCA) in COVID area [2,6]. Follow IAP Basic Life Support algorithm (Fig. 1).

- In non-COVID critical area, HCWs should preferably use full PPE or at least double gloves, eye gear and N 95 respirator for IHCA.

- Severe acute respiratory infection (SARI) or influenza like illness (ILI) cases, pending laboratory confirmation should be managed as potentially COVID-19 cases [7].
- Shield patient's mouth and nose with aerosol box (Fig. 2) / plastic drape or surgical mask before starting chest compressions if airway is not secure (Table 1).

- Do not give mouth-to-mouth or mouth-to-mask ventilation. Use compressions- only CPR until the victim's airway is secured.

- CPR is highly aerosol generating procedure though spread of aerosol can be limited by use of shields (like face mask) on airway of the victim. In view of pandemic status of COVID-19, any victim of sudden cardiac arrest even in out of hospital setting is likely to be a source of infection. While providing CPR in Out of Hospital Cardiac Arrest (OHCA) surgical mask or other airway shield may not be available. One may use home-made face mask or piece of cloth to shield the airway of the victim in such scenario.

\section{Securing the Patient's Airway}

- Secure the airway using tracheal tube (TT) or laryngeal mask airway (LMA). To minimize the exposure risk to HCWs, connect viral filter or heat moisture exchanger (HME) with viral filtering capacity between TT/LMA and $\mathrm{Y}$ end of ventilator tube/Bain's circuit/self-inflating bag [5].

- In OHCA or if intubation/insertion of LMA is delayed in IHCA, use bag and mask ventilation with viral filter and tight seal to minimize air leak.

\section{Restrictive Team Composition}

- Three member team making the triangle of resuscitators with add on responsibilities [3].

- Role 1: Airway (act as leader also)

- Role 2: Compressor to alternate with member 3

- Role 3: Automated external defibrillator (AED) or defibrillator/administer medication/assists

- Other team member(s) responsible for recording events or counseling should stay outside the resuscitation room or at safe distance from the site.

- HCWs above 60 y of age or having co-morbidities should not participate in active resuscitation code.

- Family or friend's presence is not recommended; instead remote monitoring and tele-communication is encouraged. 


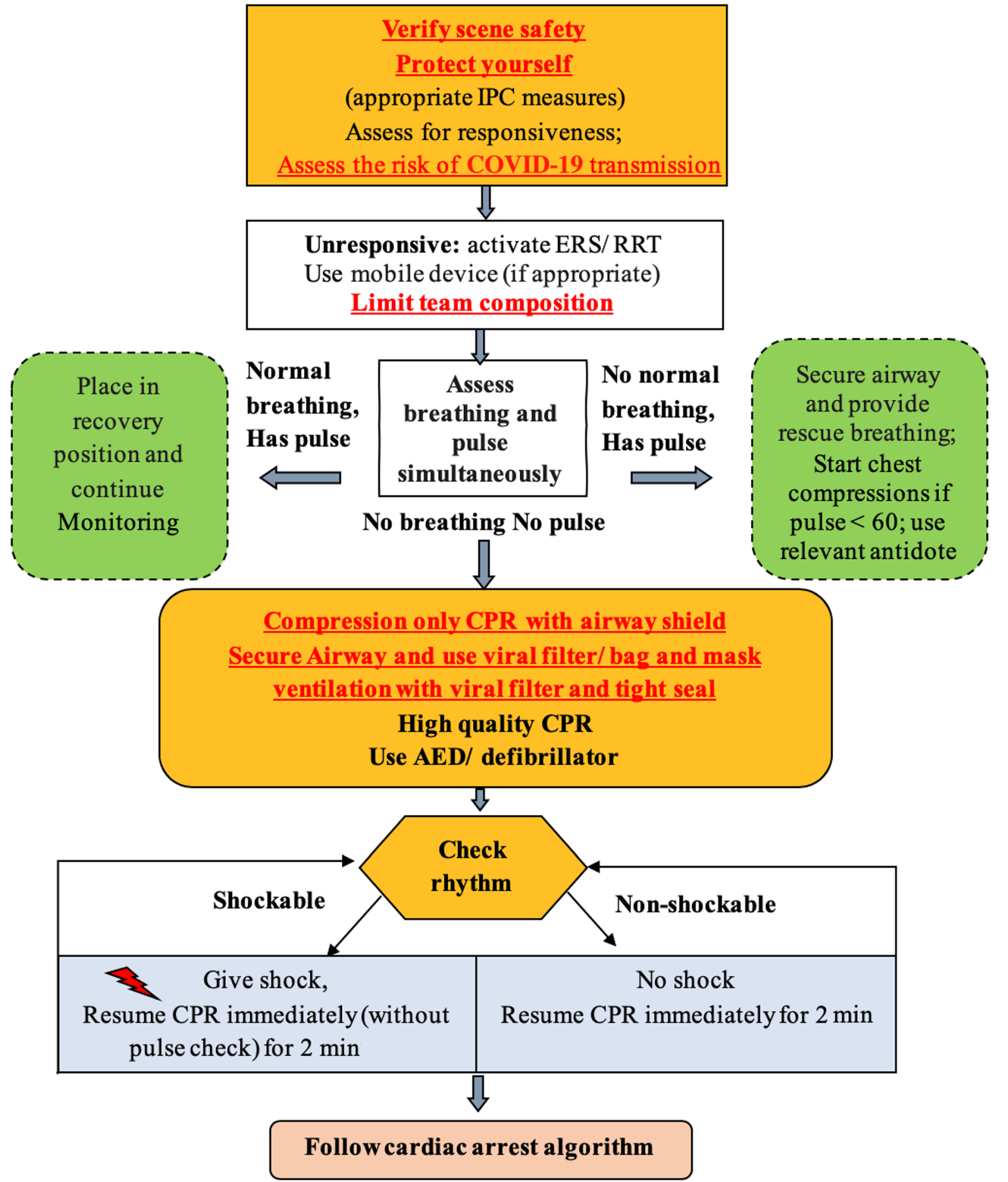

Fig. 1 IAP Basic Life Support algorithm: Updates are IAP Basic Life Support algorithm are highlighted as red and underlined text. $A E D$ Automated external defibrillator; $C P R$ Cardiopulmonary resuscitation;

ERS Emergency response system; IPC Infection prevention and control; $R R T$ Rapid response team 


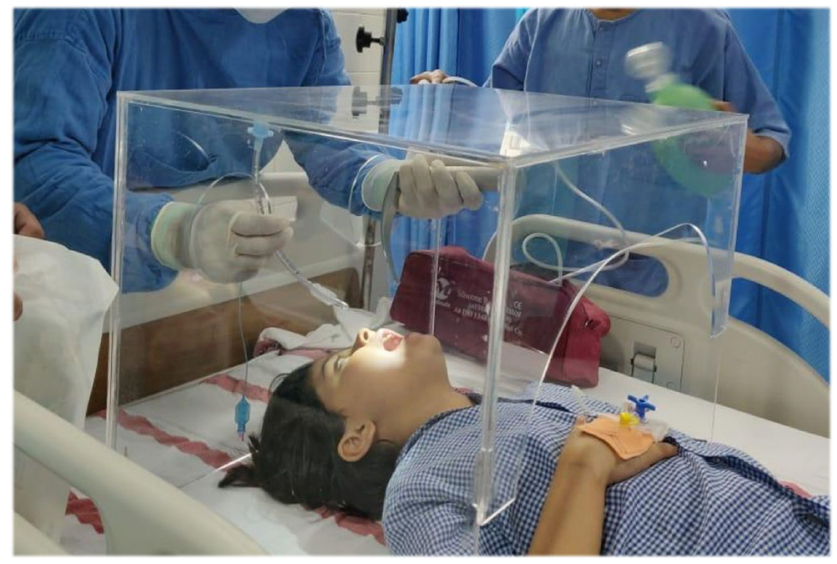

Fig. 2 Custom made aerosol box to minimize exposure during intubation

\section{Tracheal Tube Placement [8]}

- Intubate using a video laryngoscope or use LMA; or else direct laryngoscope with protective measures as mentioned above.

- Stop chest compressions while intubating or inserting LMA.

- Cuffed TTs are preferred; inflate the cuff immediately on insertion. Attach viral filter as mentioned above.

- Confirm TT placement by chest rise and/ or $\mathrm{EtCO}_{2}$ detector; avoid auscultation.

- Use closed suction system.

\section{Attach to Ventilator Early}

- Connect to a mechanical ventilator once the advanced airway is in place.

- Deliver chest compressions at a rate of $100-120$ per min without pause and breaths by ventilator at a rate of $10-12$ per min in adults/ adolescents and 12-20 per min in children [3].

- Use mechanical CPR devices, if available.

\section{AED and Defibrillator}

- Follow cardiac arrest algorithm (Fig. 3)

- Use disposable AED pads instead of defibrillator paddles to deliver shock to minimize contact with patient. In children, dose attenuated AED is preferred.

- Pads may be applied in anterior-posterior position in prone ventilated requiring CPR.

- If AED is not available, use defibrillator paddles taking infection prevention and control (IPC) precautions.

\section{CPR in Prone Position}

- Perform CPR in prone position in patients already receiving prone ventilation [9].

Table 1 Composition of crash cart

Essential items in crash cart

PPE for providers (keep at least 4 sets)

1. Non-permeable head-to-toe coveralls

2. Goggles and face-shield that covers the side of the face

3. N95 mask

4. Splash gown/apron

5. Double gloves

6. Plastic or rubber footwear

Protective items addition to standard list

1. Face shield/ surgical mask for victim

2. Aerosol box/plastic drape to cover face till advance airway in place Drugs
1. Adrenaline
2. Adenosine
3. Amiodarone
4. Atropine
5. Magnesium Sulphate $\left(\mathrm{MgSO}_{4}\right)$
6. Lidocaine
7. Midazolam, Fentanyl, Vecuronium
8. Saline flush, $5 \%$ and $10 \%$ Dextrose

\author{
Equipment \\ 1. CPR Board \\ 2. LMA/Video laryngoscope/Laryngoscope with blade \\ 3. Stylet or bougie \\ 4. Appropriate size cuffed ETT \\ 3. $2 \mathrm{ml}$ syringe for ETT cuff inflation \\ 6. Viral filter/HME with viral filter \\ 7. Bag-valve device \\ 8. Self-inflating/ flow-inflating bag/ Bains circuit \\ 9. Closed circuit system suction device \\ 10. AED/ Defibrillator with pads \\ 11. Canulae of different size \\ 12. Intraosseous needle \\ 13. Adhesive tape \\ 14. Syringes and infusion sets \\ 15. Stethoscope \\ 16. Pulse Oximeter \\ 17. BP apparatus \\ 18. $\mathrm{EtCO}_{2}$ monitor (desirable) \\ 19. Surgical airway kit (desirable)
}

$A E D$ Automated external defibrillator, $C P R$ Cardiopulmonary resuscitation, ETT Endotracheal tube; HME Heat moisture exchanger, $L M A$ Laryngeal mask airway, $P P E$ Personal protective equipment

*additional items during COVID-19 pandemic are in italics 


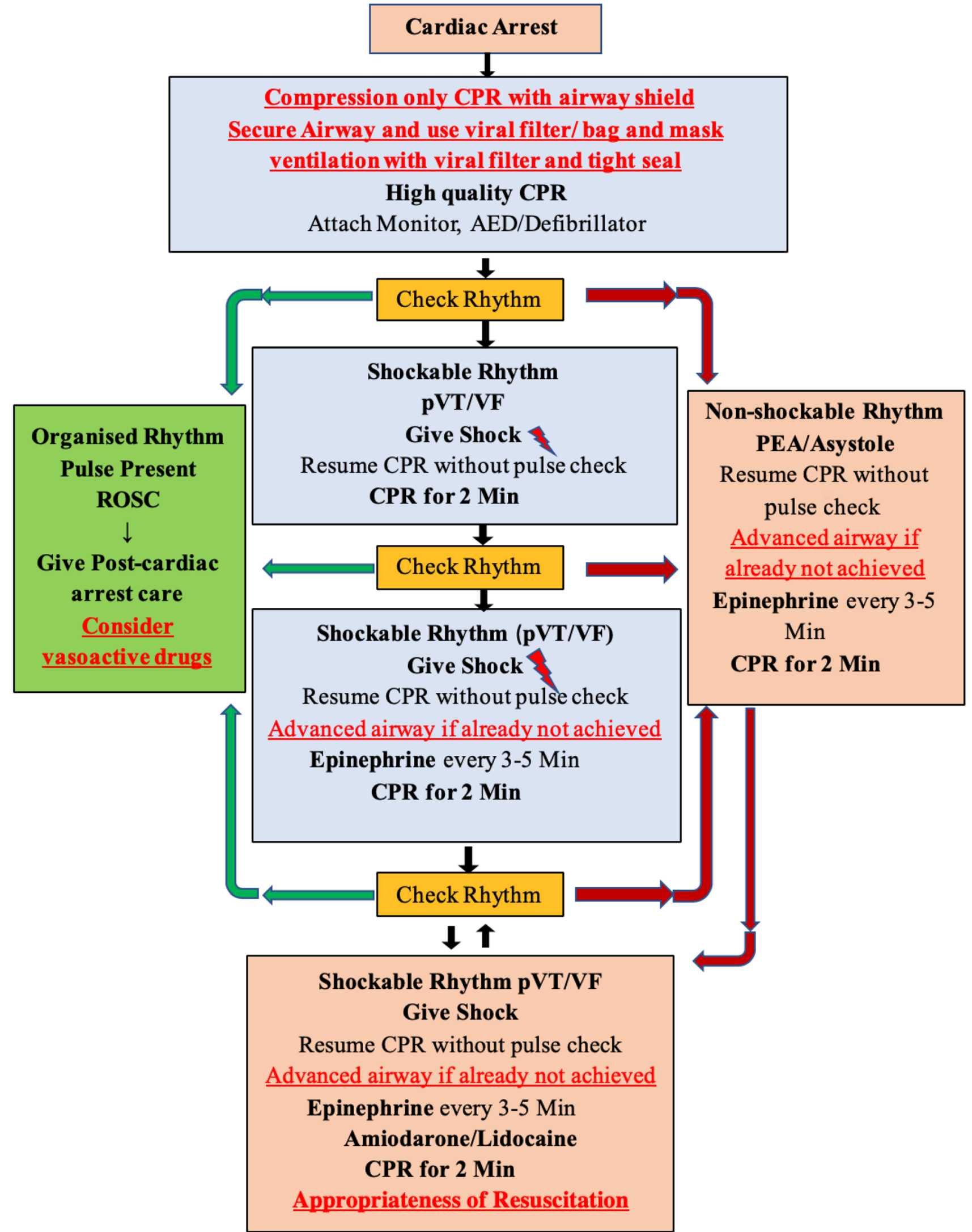

Fig. 3 IAP Cardiac Arrest algorithm: Updates in cardiac arrest algorithm are highlighted as red and underlined text. $A E D$ Automated external defibrillator; $B P$ Blood pressure; $C P R$ Cardiopulmonary resuscitation;
$I O$ Intraosseous; IV Intravenous; PEA Pulseless electrical activity; $p V T$ Pulseless ventricular tachycardia; $V F$ Ventricular fibrillation 
- Position hand just below the subscapular line over T7 to T10 vertebra on the midline. Deliver compression at the same rate (100-120 per min) and force (sufficient enough to compress one-third of antero-posterior diameter of the chest) as in the supine position.

\section{Hypomagnesemia: The 7th " $\mathrm{H}$ "}

- Hypomagnesemia is an important correctable cause of ventricular arrhythmias in COVID-19 cases. Hydroxychloroquine and/or azithromycin use also predisposes these patients to ventricular arrhythmias like Torsades de Pointes [10].

- Consider magnesium-sulfate $\left(\mathrm{MgSO}_{4}\right)$ infusion during resuscitation as seventh $\mathrm{H}$ in list of $\mathrm{Hs}$ and Ts.

\section{Early Use of Vasoactive Drugs}

- Once return of spontaneous circulation (ROSC) is achieved, consider early use of vasoactive drugs for persisting shock.

- Avoid aggressive fluid resuscitation [2].

\section{Appropriateness of Resuscitation and Duration of CPR}

- Follow your hospital policy on CPR and patient transfer.

- In patients with advance stage of disease and those with co-morbidities, possible outcome may be considered while deciding continuation or termination of CPR [11]. Risk stratification should be discussed among HCW's and communicated to parents or proxy.

\section{Summary}

These modifications are primarily targeted to ensure safety to health care worker without compromising on quality of CPR. Summary of recommendations is highlighted in Box 2. Simulation and repeated team practice is highly recommended for acquiring these new psychomotor skills.
Box 2. Summary of recommendations

Ten recommendations on CPR

1. Protect yourself before starting chest compression: P-C-A-B Sequence

2. Secure the airway to minimize the exposure risk

3. Restrict team composition to triangle of resuscitators

4. Practice additional care while placement of tracheal tube

5. Attach to ventilator early

6. Preferably use AED, if defibrillator pads are available, use pads in place of conventional paddles.

7. Consider a 7th "H", Hypomagnesemia:

8. CPR can be given in prone position with an advanced airway in place.

9. Early use of vasoactive drugs instead of aggressive fluid resuscitation in patients with ROSC .

10. Consider appropriateness of starting and duration of CPR

$A E D$ Automated external defibrillator; $C P R$ Cardiopulmonary resuscitation; ROSC Return of spontaneous circulation

Author's Contributions LT and LNT planned the update, and did literature search. LT and SG prepared the initial draft. All members of the group participated in developing consensus expert opinion over multiple written communications and video conferencing. Indian Academy of Pediatrics Advanced Life Support Basic Life Support (IAP ALS BLS) group endorsed the update.

\section{Compliance with Ethical Standards}

Conflict of Interest None.

\section{References}

1. Centers for Disease Control and Prevention. Information for Clinicians on Therapeutic Options for COVID-19 Patients. Updated April 7, 2020. Available at: https://www.cdc.gov/ coronavirus/2019- ncov/hcp/therapeutic-options.html/ Accessed 10 May 2020.

2. COVID -19 Outbreak. Guidelines for Setting up Isolation Facility/ Ward. National Centre for Disease Control, Directorate General of Health Services, Ministry of Health and Family Welfare India. Available at: https://ncdc.gov.in/showfile.php?lid=503. Accessed 10 May 2020.

3. Taneja LN, Tiwari L. BLS for professionals. 3rd ed. New Delhi: Indian Academy of Pediatrics ALS BLS Group; 2018.

4. Jayashree M, Kulgod V, Sharma AK. IAP ALS Handbook, 1st. IAP National Publication House New Delhi: Indian Academy of Pediatrics; 2018.

5. Edelson DP, Sasson C, Chan PS, et al. Interim guidance for basic and advanced life support in adults, children, and neonates with suspected or confirmed COVID-19: from the emergency cardiovascular care committee and get with the guidelines-resuscitation adult and pediatric task forces of the American Heart Association. Circulation. 2020;141:e933-43. https://doi.org/10.1161/ CIRCULATIONAHA.120.047463.

6. Rational Use of Personal Protective Equipment for Coronavirus Disease (COVID-19) and Considerations During Severe Shortages: Interim Guidance, 6 April 2020. Available at https:// apps.who.int/iris/handle/10665/331695. Accessed 10 May 2020. 
7. Ministry of Health \& Family Welfare, Govt of India. Directorate General of Health Services EMR Division. Guidance Document on Appropriate Management of Suspect/Confirmed Cases of COVID19, 07 April 2020.

8. ECRI Institute. Mechanical Ventilation of SARS Patients: Lessons from the 2003 SARS Outbreak. Health Devices, 2020.

9. Mazer SP, Weisfeldt M, Bai D, et al. Reverse CPR: a pilot study of CPR in the prone position. Resuscitation. 2003;57:279-85.
10. Roden DM, Harrington RA, Poppas A, Russo AM. Considerations for drug interactions on QTc in exploratory COVID-19 (coronavirus disease 2019) treatment. Circulation. 2020.

11. Indian Council of Medical Research Expert Group on DNAR. ICMR consensus guidelines on 'do not attempt resuscitation'. Indian J Med Res. 2020;151:303-10.

Publisher's Note Springer Nature remains neutral with regard to jurisdictional claims in published maps and institutional affiliations. 